\title{
Corela
}

Cognition, représentation, langage

HS-8 | 2010

L'interpellation

\section{Interpellativité et appellativité. Une étude pragmatique de l'avis de décès}

\section{Françoise Hammer}

\section{(2) OpenEdition}

\section{Journals}

\section{Édition électronique}

URL : http://journals.openedition.org/corela/754

DOI : $10.4000 /$ corela.754

ISSN : 1638-573X

\section{Éditeur}

Cercle linguistique du Centre et de I'Ouest - CerLICO

\section{Référence électronique}

Françoise Hammer, «Interpellativité et appellativité. Une étude pragmatique de l'avis de décès », Corela [En ligne], HS-8 | 2010, mis en ligne le 01 octobre 2010, consulté le 19 avril 2019. URL : http:// journals.openedition.org/corela/754; DOI : 10.4000/corela.754

Ce document a été généré automatiquement le 19 avril 2019

\section{(c) (i) (2)(2)}

Corela - cognition, représentation, langage est mis à disposition selon les termes de la licence Creative Commons Attribution - Pas d'Utilisation Commerciale - Partage dans les Mêmes Conditions 4.0 International. 


\title{
Interpellativité et appellativité. Une étude pragmatique de l'avis de décès
}

\author{
Françoise Hammer
}

\section{Présentation}

1 Comment identifier l'interpellation et en circonscrire le champ dans l'espace discursif en face d'autres concepts comme l'apostrophe, l'insulte, la convocation, l'invocation, l'évocation ou simplement l'interrogation et l'appel? Le flou terminologique, comme l'ont montré Détrie et Pop (ici-même) rend la tâche difficile. L'approche pragmatique choisie se propose d'aborder le fait interpellatif à partir de l'étude empirique d'un texte utilitaire, spécifique par sa situation de communication, son contexte d'énonciation et sa plurilogalité: l'avis de décès. Souvent interrogé dans le cadre d'analyses textuelles et phraséologiques, ce texte n'a guère retenu l'attention des chercheurs pour son appellativité bien qu'il interpelle son lecteur, le convoque à une réaction émotionnelle et soit, pour cette raison, un des articles les plus consultés de la presse régionale (Link, 2001).

2 L'analyse part de l'idée que toute énonciation est dialogique (Bakhtine, 1977, 134), le dialogue face-à-face, n'étant qu'une forme d'échange spécifique. "L'indice substantiel (constitutif) de l'énoncé, c'est le fait qu'il s'adresse à quelqu'un, qu'il est tourné vers l'allocutaire.» (Bakhtine 1979, 303) et qu'inversement, «la compréhension de tout énoncé est dialogique. » (Bakhtine 1979, 335) et que d'autre part, l'expression de tout acte de langage dépend à la fois de sa finalité et de son contexte d'énonciation. ${ }^{1}$ L'avis de décès ainsi considéré, constitue une intervention responsive dans la mesure où l'énonciation de $A$ appelle une réponse de $B$. Si les mécanismes linguistiques sont conditionnés par les espaces socio-culturels de leur énonciation (Pop, 2000), le domaine de l'interpellation devrait pouvoir se définir par contraste pragmatico-fonctionnel avec d'autres configurations du champ appellatif ou allocutif (Détrie, 2006) auquel elle appartient. 
L'objectif est donc d'approcher de plus près l'interpellation à l'intérieur du champ appellatif, par un examen contrastif des modalités appellatives de quatre types d'avis de décès. ${ }^{2}$ L'attention portera plus particulièrement sur les modalités d'évocation, de convocation et d'invocation.

\section{Cadre d'analyse}

\subsection{Interpellation}

3 L'approche du fait interpellatif se heurte à deux obstacles majeurs : l'espace appellatif est hétérogène et mal défini, sert le plus souvent de fourre-tout à ce qui transgresse la norme au nom de l'émotionnalité, de l'affectivité ou de l'expressivité - Pop parle dans ce sens de flou interpellatif - et les investigations partielles dont il a fait l'objet relèvent de différents domaines de recherche. La définition du terme interpeller relevée sur internet (mars 2008) fait apparaître trois acceptions dominantes :

- appel lancé par une personne à une autre, le plus souvent par un agent de la fonction publique en vue d'une arrestation (Détrie et Pop, ici-même),

- appel lancé à une assemblée représentative en vue d'information, dans un cadre contradictoire (Facq-Wellet, ici-même),

- appel à une réaction émotionnelle déclenchée par des propos inattendus et généralement contraires aux préconstruits du lecteur (Guilbert, ici-même). ${ }^{3}$

Ces trois acceptions (qui correspondent approximativement aux définitions du TFLI) ont en commun, malgré leurs divergences, de marquer une interruption (sens ancien d'interpeller), une rupture, une interrogation qui demande réponse. Les deux premières considèrent la perspective du locuteur, la troisième la réception du message par l'allocutaire. Dans l'échange verbal, les deux premières représentent des interventions d'ouverture et portent nécessairement un marquage lexico-syntaxique. L'apostrophe « apparait comme le signe le plus évident de l'allocation » (Détrie, 2006, 5). La troisième par contre dépend essentiellement de l'interprétation subjective de l'énoncé par un allocutaire auto-désigné, et n'implique donc pas de marquage linguistique. ${ }^{4}$ Tandis que les deux premières font l'objet d'études socio-linguistiques (insulte ou termes d'adresse) ou grammaticales (détachement, apposition), la troisième intègre l'analyse des préconstruits discursifs (Paveau, 2007).

5 Doit-on partant, considérer deux formes d'interpellativité, l'une marquée sur le plan phrastique et correspondant à des actes ou semi-actes de parole, spécifiques des interactions directes, l'autre non (nécessairement) marquée sur le plan phrastique, correspondant à des macro-actes et qui serait prototypique d'interactions différées?

\subsection{Le champ interpellatoire}

L'examen pragmatique du champ interpellatoire peut prendre appui sur les enquêtes du champ vexatoire mitoyen et souvent co-occurrent. Hammer (à par. 2) différencie ainsi une vexation ouverte marquée, celle de l'injure, et une vexation couverte non-marquée, celle de l'insulte ressentie (comme derrière un surcroît de politesse). Dans son analyse des configurations de l'injure, Évelyne Larguèche (à par.) part de considérations similaires et distignue quatre intervenants : « l'injurieur qui parle, émet, prononce l'injure, l'injuriaire, celui à qui s'adresse l'injurieur, l'injurié, celui dont il est question dans les propos de et le 
témoin, c'est-à-dire celui qui assiste à la situation d'injure, écoute les propos échangés ou encore les lit » et dont elle souligne l'importance dans le cas d'injure interpellative « dont l'objectif est moins d'injurier que de convaincre le témoin.». Le statut et le nombre des intervenants permet dès lors de délimiter les configurations injuriaires en fonction des situations de communication. Une approche similaire semble applicable pour différencier l'interpellation de fonctions appellatives proches telles que: évocation, invocation ou convocation. À l'exemple de Beaumatin (1995) le terme témoin-prétexte désignera le destinataire non-allocuté et témoin-interprète l'observateur extérieur.

Une première approche pragmatique pourrait partir du schéma suivant :

Tableau 1. Interpellativité

\begin{tabular}{|l|l|l|}
\hline & ouverte & couverte \\
\hline point de vue & locuteur-interpellateur & témoin-interprète \\
\hline marquage & explicite & implicite ou a-spécifique \\
\hline interaction & directe & virtuelle, auto-désignée \\
\hline
\end{tabular}

7 Qu'elle soit ouverte ou couverte, l'interpellation implique, comme l'injure sa reconnaissance comme telle par l'allocutaire auto-désigné ou non. Elle est donc le produit d'une interaction verbale explicite ou implicite. Contrairement à l'injure cependant, la parole interpellative n'est pas per se un acte langagier à finalité spécifique, elle peut viser des réactions pragmatiques multiples, dont l'injure. Il est possible, dit (Larguèche, à par.), «d'injurier un absent en l'interpellant». Elle ne constitue qu'une incitation à une réaction de l'allocutaire en dépendance avec le contexte d'énonciation. Il convient donc de dissocier moule lexico-syntaxique et potentialité pragmatique. Le moule lexicosyntaxique de l'interpellation ouverte concrétise la jonction des fonctions phatique et conative (Jakobson, 1960). La fonction phatique assure le positionnement interpersonnel des interactants, leur prise de contact est assumée par une formule interjective (interjection et/ou terme d'adresse) anté- ou postposée à une proposition conative destinée à déclencher une réaction du destinataire : injonctions, vocatifs, relationèmes et autres séquences à fonction appellative. Quelques exemples de L'avare de Molière le montrent. ${ }^{5}$

[1a] Cléante. - Ah ! traître que tu es, où t'es-tu donc allé fourrer ? Ne t'avais-je pas donné ordre [...] ? (acte II, scène 1)

[1b] Frosine. - Hé ! c'est toi, mon pauvre La Flèche ! D'où vient cette rencontre?

La Flèche. - Ah ! ah! c'est toi Frosine ! Que viens-tu faire ici ? (acte II, scène 4)

[1c] Maître Jacques. — Eh! eh! eh! messieurs, qu'est ceci ? à quoi songez-vous ?

(acte IV, scène 4)

8 L'intervention interpellative ouverte ou apostrophe, phatique et conative, intègre dans son énonciation, par le choix des termes d'adresse employés, la réaction attendue de l'allocutaire, «la façon d'interpeller étant totalement significative non seulement du rapport projeté, mais aussi de la réaction supposée d'autrui à la construction de ce rapport » (Détrie, 2006, 8). ${ }^{6}$ Elle se fonde sur un positionnement interpersonnel défini des intervenants. L'analyse de l'avis de décès et de ses variantes tente dans cette voie, de dégager les mécanismes linguistiques employés par ses annonceurs selon les rapports 
sociaux projetés et impliqués avec les allocutaires. Le texte présente un enjeu social auquel doit s'accorder la rhétorique appellative.

\subsection{Avis de décès}

9 Dans sa classification des textes utilitaires Gebrauchstextsorten Rolfs (1993, 173) range l'avis de décès au nombre des textes admissifs, c'est-à-dire conçus de façon primaire en fonction de l'allocutaire visé. ${ }^{7}$ Avis, annonce, faire-part sont des textes dialogiques qui fournissent à leurs destinataires avec l'information des instructions pratiques. L'avis de décès a en ce sens une fonction appellative évidente Ses désignations elles-mêmes l'indiquent. Son objectif sur le plan social vise d'abord à resserrer les liens de la communauté conviée au partage de la douleur avec la famille en deuil, il notifie aussi le respect des conventions sociales et du souvenir du défunt. L'expression langagière du texte est ainsi subordonnée à la fonction de la communication aux conventions sociales ainsi qu'à des facteurs textuels (Pop, 2000, 139-143) et éditoriaux. «[...] La situation et l'auditoire contraignent le discours intérieur à s'actualiser en une expression extérieure définie, qui s'insère directement dans le contexte inexprimé de la vie courante» (Bakhtine, 1977, 138). L'avis de décès des DNA et du RL se définit (Hammer, à par. 1) par une typographie spécifique. Il s'inscrit dans un encadré noir, un symbole d'appartenance religieuse ou un portrait du défunt précédant le plus souvent l'assertion de la mort, constitutive du texte. Il s'agit d'un message public, émanant d'un groupe d'énonciateurs hétérogène à l'adresse d'interlocuteurs indifférenciés, présumés prêts à y répondre. Les conventions sociales ont longtemps exigé, sur la base d'une opposition privé-public, une retenue dans l'expression émotionnelle et les marques d'appellativité. L'exiguïté de l'espace scriptural à l'intérieur du cadre noir impose des formulations elliptiques et stéréotypes. Le caractère officiel du texte, l'usage de la langue de distance (Lüger \& Schäfer, 2004) et l'évitement des marques de proximité font préférer à l'interpellation directe un marquage sémantico-pragmatique indirect: assertions directives et intimations implicites. La multiplicité des instances appellatrices (locuteur, énonciateurs-annonceurs) et réceptrices (parents, amis, relations, récepteurs autodésignés), déterminante pour les modalités d'interaction appellatives, se trouve ainsi masquée par le nivellement stylistique d'une formulation qui se veut neutre et objectivante. L'appellativité du texte a été de ce fait, longtemps négligée. L'évolution actuelle (Linke, 2001 ; von Lage-Müller, 1995 ; Hammer, à par. 1) vers une communication individualisée et une propension à l'expression de sentiments personnels, introduit dans le texte une diversification qui permet une investigation plus différenciée des interactions appellatives qui en fait le sous-tendent et de leurs modalités.

Dans le corpus analysé, composé uniquement d'annonces privées, publiées par la famille ou les proches du défunt, on examinera ici quatre groupes d'avis appelés ici : réductif, générique, extensif et épistolaire dont on tentera de décrire les modalités appellatives. L'intérêt porte essentiellement sur trois d'entre elles, l'évocation, la convocation et l'invocation et leurs relations avec l'interpellation dans le champ appellatif. 


\section{3. Étude empirique}

\subsection{L'avis réductif}

11 Le type réductif se limite aux composantes constitutives du texte : l'assertion du décès, suivie des nom, prénom et éventuellement dates de naissance et de décès du défunt. La localisation du texte dans la rubrique mortuaire et le cadre noir constituent les seules marques appellatives. Son mandataire n'étant pas mentionné, l'assertion prend la forme : On est prié d'annoncer ou M. X est décédé. Message différé, adressé à un allocutaire indifférencié par un locuteur non-identifié, le texte présente un potentiel appellatif minimum. En l'absence d'identification et de positionnement relationnel des interactants, le texte, réduit à une simple assertion informative, exclut toute relation interpersonnelle. Son appellativité couverte ne peut résulter que de facteurs extra-linguistiques, liés à la personnalité du témoin-interprète, ses souvenirs et ses rapports avec le défunt ou l'annonceur présumé. Elle relève de l'évocation «action de rappeler à la mémoire, de remettre sous les yeux ce qui a disparu » (Dictionnaire de l'académie française, 1935). Elle se rapproche de l' interpellation pragmatique (Guilbert ici même) sans impliquer toutefois de prise de position distanciatrice sur un dire.

12 L'avis réductif est rare dans le corpus et les autres journaux de la presse régionale (RL et Ouest-France). Ainsi même un message lapidaire et distant, tente de donner à son information une présentation personnelle à l'adresse du récepteur comme le montre l'intervention factice d'un locuteur individualisé par Nous et les remerciements éventuels (figure 1). L'évocation représente une forme d'appellativité interpersonnelle minimale nécessaire pour le maintien des liens communautaires auquel le texte réductif répond.

Figure 1

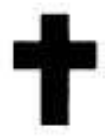

\section{Monsieur Antoine NONNENMACHER}

nous a quittés le 30 juin 2006.

Selon sa volonté, la cérémonie religieuse a été célébrée dans l'intimité.

Nous remercions tous ceux qui l'ont soutenu durant cette épreuve.

o26430.

Vendenheim, le 9 juillet 2006.

La famille en deuil. 


\subsection{L'avis générique}

13 L'avis générique, prototypique des DNA (figure 2) comprend en plus de l'information du décès, des indications pratiques à l'ordre des récepteurs. Ses annonciateurs sont nommés ou identifiables par leurs relations avec le défunt.

Figure 2

CET AVIS TIENT LIEU DE FAIRE-PART

ET DE REMERCIEMENTS

Nous avons la douleur de faire part du décès de

\section{Monsieur Charles DOERFLINGER}

notre très cher père, beau-père, grand-papa, frère, beau-frère, parrain. oncle, compagnon, parent et ami, enlevé à notre affection le 4 mars 2008 , dans sa $90^{\circ}$ annèe.

La cérémonie religieuse aura lieu le vendredi 7 mars 2008 ,

à $10 \mathrm{~h} 30$, en l'église des Chaines de Sainte-Marie-aux-Mines.

Selon sa volontè, son corps sera incinèré.

68160 Sainte-Marie-aux-Mines

Les familles en deuil:

DOERFLINGER - ROSENZWEIG - HOSTETTER

14 L'emploi de relationnels, le nom des annonceurs, les formulations émotionnelles (son papy adoré, son très cher père), les directives pragmatiques (la cérémonie aura lieu), le métatexte ( cet avis tient lieu de faire-part et de remerciements) et l'adresse de contact des annonceurs témoignent de sa visée appellative. L'adjonction récente de remerciements personnalisés à l'intention des aides des derniers moments (figure 3) souligne une individualisation appellative croissante du message.

15 Caractéristique de l'avis prototypique est le dédoublement du groupe scripteurannonceurs et la multiplicité des allocutaires qui en font un lieu d'interactions plurielles schématisées comme suit :

Tableau 2.

\begin{tabular}{|l|l|}
\hline locuteur & allocutaire \\
\hline scripteur & indifférencié \\
\hline \multirow{3}{*}{ annonceur } & cité \\
\cline { 2 - 2 } & différencié \\
\cline { 2 - 2 } & indifférencié \\
\hline
\end{tabular}

16 Le scripteur anonyme (formulaire du journal ou des pompes funèbres) assume la fonction d'informateur intermédiaire neutre comme l'indiquent les formulations impersonnelles : "On nous prie d'annoncer le décès de X de la part de ». Sa présence le plus souvent occultée, transparaît parfois dans certaines incohérences textuelles : 
[2] M. Christian Mallo, M. Jacky Mallo, ses fils ont l'immense tristesse de faire part du décès subit de Madame Robert Mallo, leur très chère maman, cousine, parente et amie.

Sa seule fonction réside dans la transmission a-personnelle d'un message à un allocutaire indifférencié. L'annonceur, commanditaire du message, est lui-même un être multiple, uni par des relations familiales et sociales avec le défunt: «mon époux, mon ami, mon confident, grand père attentionné, papy, papapa, pépé » ou «mon cher et inoubliable époux, notre très cher papa, papy et beau-père, beau-frère, oncle, cousin, parent et ami " qui justifient son intervention. Le vous de "Nous avons la douleur de vous faire part " s'adresse à des allocutaires pluriels, indifférenciés, plus ou moins proches du défunt et des annonceurs. Les remerciements du métatexte : « cet avis tient lieu de faire-part - et de remerciements - » vont à deux groupes différents, des remerciés cités, pour une action effectuée et des remerciés indifférenciés : tous ceux qui, toutes les personnes qui, pour une démonstration d'empathie anticipée :

[3] Nous remercions Dominique Moog, curé de notre paroisse, pour son accompagnement, le docteur Heimig, son médecin traitant, et les infirmières Estelle, Michelle et Véronique pour leur gentillesse et leur dévouement ainsi que toutes les personnes qui s'associent à notre très grand chagrin et à notre espérance. (DNA 2006-06-28).

18 L'objectif d'une telle scénographie (Maingueneau, 1996), est de toute évidence, de consolider les liens sociaux. Elle ne tend pas à une interaction interpersonnelle. La pluralité allocutive implique une stratégie de l'évitement (Kerbrat-Orecchioni, 1992) et un marquage appellatif indirect par des assertions directives : « La cérémonie religieuse aura lieu le samedi 15 juillet 2006 à 14 h 30, en l'église Sainte-Pierre-et-Paul de Hochfelden, où on se réunira." ou des injonctions elliptiques: "Ni fleurs ni couronnes", "Pas de plaques, messes et fleurs naturelles ", " Prière de s'abstenir de visites de condoléances ", « registre de condoléances ». L'avis de décès prototypique ne présente pas de marquage phatique interpersonnel. La communication entre un locuteur pluriel la famille en deuil et un allocutaire multiple semble exclure l'établissement de relations interpersonnelles. Ces observations semblent en faveur d'une restriction du concept d'interpellation à une interaction verbale interpersonnelle entre deux interlocuteurs statutairement définis. Pour contourner cet écueil, l'insulte recourt à des regroupements singularisants : bande de ou espèce de. L'avis générique est ainsi un appel adressé à un allocutaire multiple et hétérogène par un groupe spécifié mais lui-même multiple. La modalité appellative employée relève plus de la convocation que de l'interpellation, le domaine préférentiel de celle-ci étant celui de relations binaires, où locuteur et allocutaire occupent un statut défini. L'examen du texte générique justifie la position de Détrie $(2006,8)$ pour qui « interpeller, c'est [...] construire une sphère interpersonnelle au sein de laquelle l'instance d'énonciation prédique non seulement la présence d'autrui, mais aussi son positionnement en tant que coénonciateur ». Dans ce contexte, les remerciements au personnel soignant, (désigné par nom et titre pour les docteurs et prénoms pour les aides, plus proches de la famille) peuvent prendre une fonction appellative couverte, intensifiant l'empathie de l'allocutaire pour le défunt ou sa famille.

\subsection{L'avis extensif}




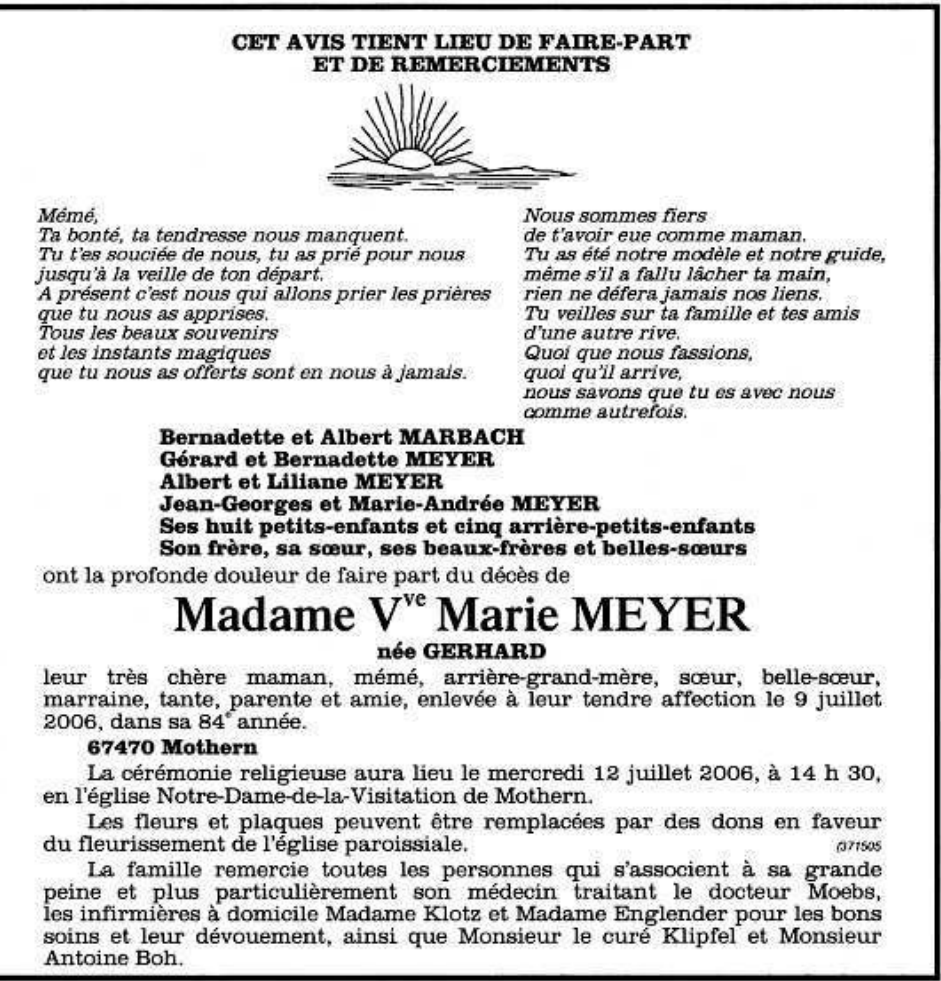

L'avis extensif ( $25 \%$ des annonces des DNA) complète le scénario générique d'un prétexte (motto, épigraphe, citation littéraire, biblique ou autre écrit de circonstance) qui prend généralement la forme d'une apostrophe (figure 3).

Il introduit ainsi dans le texte d'accueil une communication dialogale entre: Dieu, le défunt, l'annonceur et le lecteur, schématisée dans figure 3 (Ringlet, 2002, 182) :

Figure 4

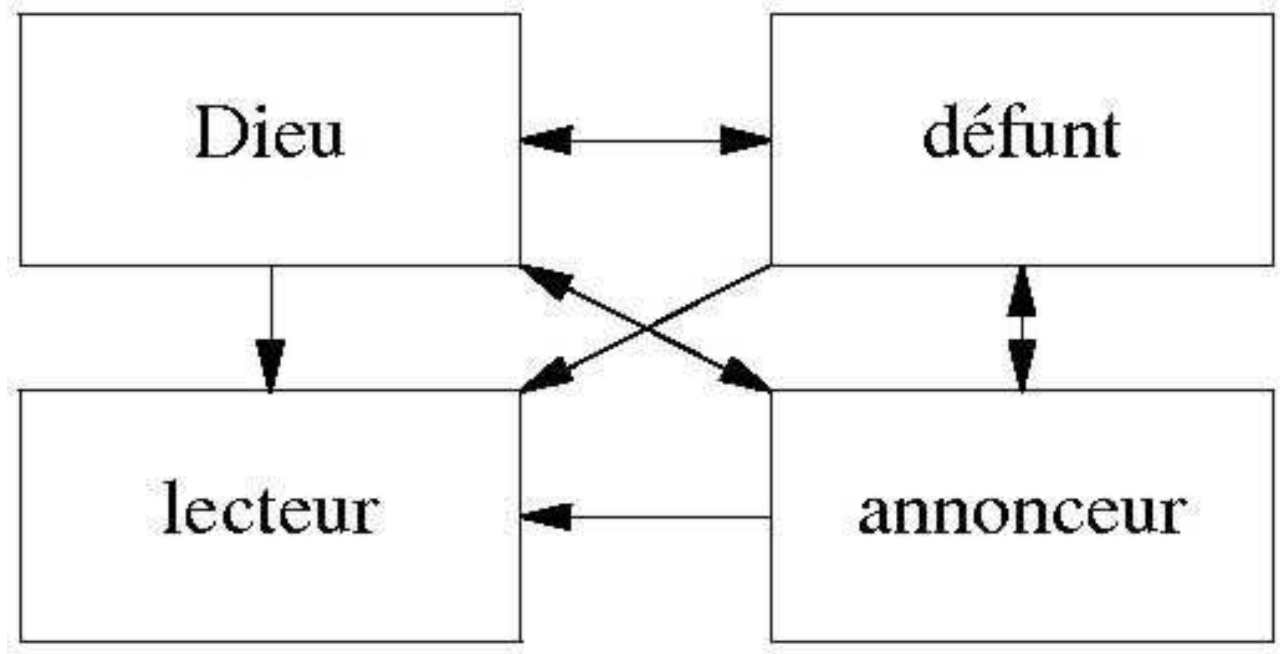

21 Les interventions des actants du pré-texte reproduisent le moule lexico-sémantique de l'interpellation, le statut respectif de ceux-ci n'y répond cependant pas. Dieu, le défunt et l'annonceur peuvent engager un dialogue virtuel, le témoin-prétexte lui, ne peut ratifier 
l'interpellation dont il fait l'objet. L'appel lancé par Dieu et le défunt s'adresse au groupe indifférencié des survivants, annonceurs et tous témoins confondus,

[4a] Arrachez-vous à vos craintes Venez à moi, vous tous qui êtes chargés et fatigués, je vous soulagerai (Mt 11,28)

[4b] Pensez à moi dans les moments de joie. Dans la main de Dieu, je serai toujours près de vous

celui de l'annonceur au groupe indifférencié de la communauté du défunt et des récepteurs :

[5] Elle aimait la vie. Vous qui vivez, pensez à elle

Un semblant de dialogue interpersonnel ne peut s'établir qu'entre annonceur et défunt ou annonceur et Dieu :

[6a]Puisqu'il faut quitter cette terre,

Permettez-moi, mon Sauveur, mon Père,

De reposer auprès de vous. (Jean 17. 23)

[6b] Je pars vers la lumière rejoindre papa et ma petite sœur,

Priez le seigneur pour moi. Il est très bon.

[6c] Oh! Maman, au ciel je te retrouverai

Vole, chante et danse dans un ciel de liberté

[6d] Jésus, si tu n'es pas un leurre [...] supplée donc à leur impuissance.

Le pré-texte introduit ainsi une simulation d'interaction dans le but de transcender la mort et de maintenir par delà le décès, l'unité de la communauté. D'où les formules : "Mais ce n'est qu'un au revoir. Car un jour on se retrouvera. ». L'apostrophe du motto est l'expression d'une invocation, d'un souhait ou d'une prière où expressivité et appellativité se tiennent la balance. ${ }^{8}$ ''apostrophe ne prévoit dans son énonciation aucune réplique ratificatrice. Elle vise à caractériser le défunt comme les annonceurs et à apporter un réconfort au récepteur dans le cadre des conventions sociales établies. Elle peut tout au plus avoir un effet interpellatif couvert sur le récepteur en fonction de connaissances et de préconstruits extra-linguistiques.

\subsection{L'avis épistolaire}




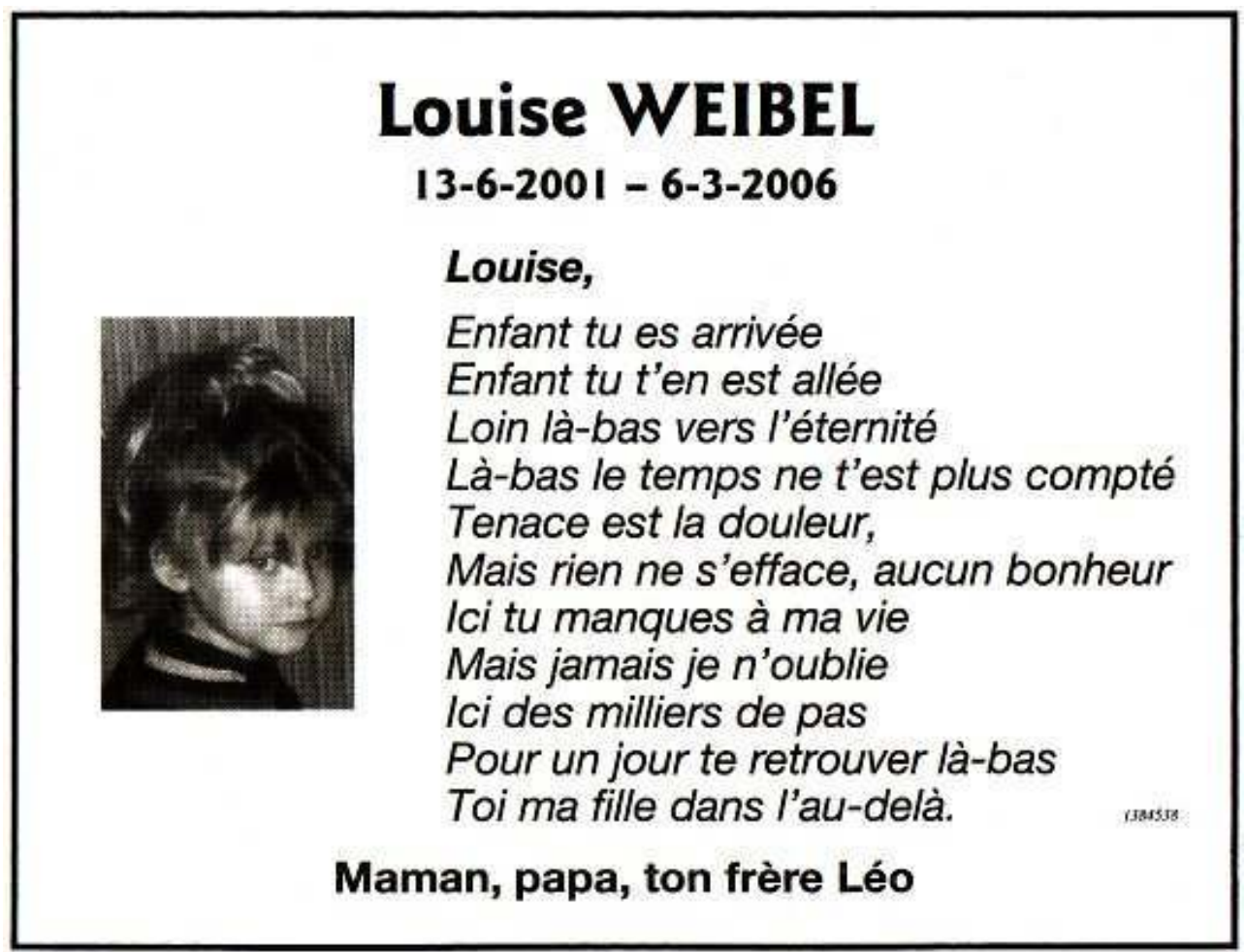

Dans ce type de message (figure 5) l'appellatif l'emporte sur l'informatif. Il y a inversion des fonctions de l'avis générique. L'avis de décès épistolaire prend la forme d'une lettre intime adressée au disparu, appelé par son prénom ou un relationème et avec lequel l'annonceur entretient un dialogue fictif. Il se rapproche donc sur le plan lexicosyntaxique de l'interpellation par son caractère dialogique interpersonnel. Seules des données extra-textuelles (proximité d'un avis générique) ou extra-linguistiques (liens de proximité interpersonnels) permettent d'identifier l'auteur du message. L'allocutaire direct est certes le le défunt mais le texte s'adresse en fait à des témoins-prétextes autodésignés. L'apostrophe épistolaire sert à la mise en scène de sentiments personnels, l'avis de tristesse (Traueranzeige) remplace l'avis de décès (Todesanzeige) (von Lage-Müller, 1995). La progression quantitative des avis de décès épistolaires (10\% environ dans les DNA) témoigne d'une tendance à la transgression de l'opposition privé-public (Link, 2001). D'informatif le texte devient l'expression personnelle de la douleur. Cette tendance est encore plus nette dans les avis d'anniversaire qui vont jusqu'à la déclaration d'amour : «Mon grand amour, je t'aime. Ton épouse Huguette». L'annonceur tente par l'appel lancé au défunt, de maintenir avec lui, du moins en apparence, des liens interpersonnels. La configuration infratextuelle de l'avis épistolaire répond à la condition définitoire d'interpellativité par un positionnement clair des relations interpersonnelles des interactants (annonceur et défunt nommés) et la création d'un espace d'intersubjectivité, jalonné par des marqueurs d'interpellativité : termes d'adresse, vocatifs, impératifs. Elle n'implique cependant pas de validation du destinataire. Elle relève de l'apostrophe rhétorique (Détrie, 2006, 16) et de la figuration d'interpellation. L'objectif social est d'affirmer la présence du défunt dans le monde des survivants et plus encore de positionner le locuteur au sein de la communauté : « Nous ne t'oublierons jamais. ». L'avis de décès joue le rôle d'invocation expressive, d'appel indirect à l'empathie du récepteur 
auto-désigné devant la tristesse de l'annonceur. L'incohérence de l'apostrophe et de la signature (figure 5) en sont le signe.

L'analyse empirique met ainsi en évidence dans les quatre groupes d'avis de décès, l'interaction de modalités appellatives diverses: évocation, convocation et invocation par opposition auxquelles, le fait interpellatif semble pouvoir se définir comme initiateur d'un échange verbal interpersonnel entre deux interlocuteurs spécifiés sur la base d'un statut interactionnel accepté par chacun d'eux. Un refus de ratification statutaire comme dans l'exemple suivant :

[7]Tout aurait été plus simple si M. Sarkozy avait eu le sens de la repartie de son prédécesseur, qui, un jour, alors qu'il sortait de la messe à Bornes-les-Mimosas fut ainsi interpellé :

- «Connard!

— Enchanté. Moi, c'est Jacques Chirac. » (Le Monde 2008-03-02)

rend l'objectif de l'intervention caduque. Ces observations incitent à réserver d'un point de vue fonctionnel, le terme d'interpellation à un espace d'appellativité partagée, de caractère phatique et conatif, et dont la fonction pragmatique dépend de la validation de l'allocutaire.

\section{Remarques finales}

Par delà sa fonction d'assertion, l'avis de décès répond à des fonctions d'ordre appellatif dans la mesure où il oriente l'action de l'allocutaire ou tente d'influencer son état émotionnel. L'examen des scénographies de quatre types d'annonces mortuaires met en lumière des configurations appellatives multiples, a-personnelles et interpersonnelles, polylogales ou dialogales. Par comparaison aux modalités d'évocation, de convocation et d'ìnvocations relevées, l'interpellativité semble pouvoir se définir comme comme une modalité appellative spécifique relevant d'un module d'interaction dialogale et intersubjective impliquant la ratification de l'allocutaire.

Si les apostrophes de l'avis épistolaire et le pré-texte de l'avis extensif portent des marques formelles d'interpellativité, le but du message global n'est cependant pas d'instaurer un échange dialogal interpersonnel. Les moyens lexico-syntaxiques de l'interpellation sont ici détournés vers un objectif plurirelationnel qui vise à resserrer les liens communautaires. La mise en scène de sentiments de douleur et d'affliction a pour objet de transcender la mort et de maintenir par un simulacre de dialogue, la présence du défunt dans la mémoire de la communauté allocutaire, d'affirmer l'appartenance de l'annonceur à celle-ci et d'appeler à son empathie. Le caractère épidictique du message répond aux conventions sociales du : de mortuis nihil, nisi bene.

L'interpellation ouverte suppose donc la conjonction de trois facteurs: identification phatique de l'allocutaire, intimation conative du locuteur (Benveniste, 1974, 84) et ratification responsive de l'allocutaire. Son moule lexico-syntaxique peut soutenir des objectifs pragmatiques divers, insultatoires (cf. ci-dessus) ou laudatoires : «Hé bonjour, Monsieur du Corbeau! Que vous êtes joli ! Que vous me semblez beau!» (La Fontaine : Le corbeau et le renard). L'interpellation ouverte assure ainsi au dialogue engagé ou à engager une assise interpersonnelle. Ces observations confirment l'analyse de Détrie (2006) sur l'apostrophe comme procédé d'identification existentiel à l'acte interpellatif et la thèse de Rolf (1993, 72-79) sur la nécessité de prendre en compte la fonction pragmatique du dire dans l'analyse des actes verbaux. 

l'interpellation ouverte, celle-ci ne relève pas de mécanismes lexico-syntaxiques semblables. C'est la prise de position personnelle et émotionnelle d'un allocutaire autodésigné, témoin-interprète, face à une assertion contraire à ses convictions socioculturelles, un acte réactif à une intervention interpellative supposée sur la base des préconstruits de la doxa. La réponse de Sylvain Gouguenheim (L'EXPRESS 2008-06-12, p. 58) à ses détracteurs au sujet de la polémique suscitée par son livre Aristote au MontSaint-Michel rend bien compte du phénomène.

J'ai mis en cause, sans violence, une doxa. J'ai aussi dérangé ce qu'on appelle le mandarinat. Pour les spécialistes, je ne suis pas habilité à m'exprimer sur le sujet [... ] On ne me le pardonne pas. J'ai seulement voulu m'adresser au grand public. Ce que je regrette, c'est qu'à un livre qui peut être discuté on répond par des pétitions et le lynchage médiatique.

D'un point de vue méthodologique, il semblerait donc préférable de réserver le terme d' interpellation à une forme d'appellativité marquée, délimitée par un contexte d'énonciation spécifique. Entre interpellativité couverte et appellativité la frontière semble dans la langue courante, flottante. Interpeller s'emploierait-il, pour marquer l'interrogation subjective posée par un texte, quand l'objet du verbe est animé (cf. supra) et appeler quand celui-ci est inanimé, comme dans les exemples qui suivent?

[8a] De telles phrases n'appellent pas de réponse. Mais quelques commentaires, tout de même. (Le Monde 2008-08-01)

[8b] La découverte de l'ailleurs appelle une attitude d'ouverture, de curiosité et même de générosité. (Le Monde des livres 2008-08-01).

Entre expressivité et informativité, le champ de l'appellativité et de ses modalités restent difficile à cerner.

\section{BIBLIOGRAPHIE}

Bakhtine, M. (1977). Le marxisme et la philosophie du langage. Paris : Éditions de Minuit.

Bakhtine, M. (1979). Esthétique de la création verbale. Paris : Gallimard.

Beaumatin, É. (1995). « La violence verbale. Préalables à une mise en perspective linguistique ». In É. Beaumatin \& M. Garcia (Eds.), L'invective au Moyen Age : France, Espagne, Italie. (pp. 21-35).

Paris : Presses Sorbonne Nouvelle.

Benveniste, É. (1974). Problèmes de linguistique générale. Paris: Gallimard.

Buridant, C. (Ed.) (2006). L'interjection : jeux et enjeux. Paris : Larousse.

Charaudeau, P. (2004). « La figure du tiers dans le discours publicitaire. » In P. Charaudeau (Ed.), La voix cachée du tiers. Des non-dits du discours (pp. 166-179). Paris : l'Harmattan.

Détrie, C. (2006). De la non-personne à la personne : l'apostrophe nominale. Paris : CNRS Éditions.

Eckkrammer, E. (1996). Die Todesanzeige als Spiegel kultureller Konventionen : eine kontrastive Analyse. Bonn : Romanistischer Verlag. 
Hammer, F. (à par. 1). Wieviel Liebe darf es sein ? Französische Todesanzeigen im Vergleich. In H.W. Lenk \& H.-H. Lüger (Eds.), Kontrastive Medienlinguistik. Landau : Verlag Empirische Pädagogik.

Hammer, F. (à par. 2). « Cherchez l'insulte! trouvez l'outrage! Une approche du champ vexatoire ». In communication au colloque : Les insultes en français. Université de Savoie, Chambéry (2006).

Jakobson, R. (1960). "Linguistics and Poetics". In T. A. Sebeok (Ed.), Style in language (pp. 350-377). Cambridge, Mass. : MIT.

Jeanneret, T. (1999). La coénonciation en français, approche discursive, conversationnelle et syntaxique. Bern : Peter Lang.

Kerbrat-Orecchioni, C. (1992). Les interactions verbales, tome 2. Paris : Armand Colin.

Lage-Müller, von der, K. (1995). Text und Tod. Tübingen : Niemeyer.

Larguèche, E. (à par ). «L'injure à la trace ». In communication au colloque : Les insultes en français . Université de Savoie, Chambéry (2006).

Linke, A. (2001). Trauer, „Öffentlichkeit und Intimität. Zum Wandel der Textsorte Todesanzeige.“ In U. Fix, S. Habscheid, \& J. Klein (Eds.), Zur Kulturspezifik von Textsorten (pp. 155-223). Tübingen : Stauffenburg.

Lüger, H.-H. \& Schäfer, P. (2004). „Adressatenorientierung in der deutschen und elsässischen Regionalpresse“. In C. Frenkel, H.-H. Lüger, \& St. Woltersdorff (Eds.), Deutsche und französische Medien im Wandel (pp. 31-70). Landau : Knecht.

Maingueneau, D. (1996). « Contexte et scénographie. » Scolia, 6, 185-199.

Paveau, M.-A. (2007). Les prédiscours sens, mémoire, cognition. Paris : Presses Sorbonne Nouvelle.

Pop, L. (2000). Espaces discursifs. Pour une représentation des hétérogénéités discursives. Peeters :

Louvain-Paris.

Ringlet, G. (2002). Ces chers disparus. Bruxelles : Éditions Labor.

Rolf, E. (1993). Die Funktionen der Gebrauchstextsorten. Berlin : de Gruyter.

Roulet, E. (1985). L'articulation du discours en français contemporain. Bern : Peter Lang.

\section{NOTES}

1. «Même [...] le style neutre, ou objectif, le style des exposés concentrés sur leur objet et qui semblerait ignorer l'autre, n'est pas sans impliquer une certaine idée du destinataire [...]. Ce destinataire peut être le partenaire-interlocutaire immédiat du dialogue dans la vie courante, il peut être l'ensemble différencié de spécialistes [...] il peut être l'auditoire différencié des contemporains, des condisciples, des adversaires et ennemis [...] il peut même être, de façon absolument indéterminée, l'autre non concrétisé. » (Bakhtine, 1979, 303).

2. Le corpus examiné se compose de 300 avis de décès parus en 2006 dans Les Dernières Nouvelles d'Alsace (DNA) et d'une cinquantaine d'annonces mortuaires du Républicain Lorrain (RL) (2006-2008).

3. Deux exemples : "Le Nouvel Age et l'église, interpellation réciproque », ouvrage de Guy Giroux et une citation de Merleau-Ponty (Monneret dans L'Information grammaticale $n^{\circ} 113,17$ ) : « Mais le langage parlant, c'est l'interpellation que le livre adresse au lecteur non prévenu.» (Les italiques sont de moi.) 
4. Guilbert (ici-même) parle d'interpellation pragmatique.

5. Dans l'acte d'injure les deux fonctions peuvent être assumées par un seul terme : imbécile!.

6. Pour éviter des confusions terminologiques, le terme interpersonnel est employé ici de préférence à intersubjectif.

7. Der Textproduzent nimmt an, daß der Adressat wissen möchte, wissen sollte oder wissen muß, was ihm übermittelt werden soll. (Rolfs, 1993, 179).

8. Sur les définitions des actes de parole expressifs et directifs cf. (Rolf, 1993, 73).

\section{RÉSUMÉS}

Partant de l'hypothèse générale du caractère interlocutif de tout discours, l'objectif est de contribuer à la circonscription du fait interpellatif par une analyse contrastive de divers espaces du champ appellatif. L'article se propose d'interroger à cet effet, dans une étude empirique, les structures appellatives d'un texte répondant à des conditions d'énonciation et des objectifs pragmatiques spécifiques : l'avis de décès. L'examen de quatre variantes permet ainsi de mettre en avant le caractère interpersonnel de l'illocution interpellative.

The present empirical investigation focuses on special aspects of interpellativity. In an attempt to define its scope we analyse appellative structures in four subtypes of death notices. The general hypotheses behind this study are the interlocutivity of language and the specificity of appellative patterns depending on context and purpose. In confrontation with other concepts such as convocation, invocation and evocation, interpellativity appears to be a particular form of appellativity that secures the acceptance of a dialogue at interpersonal level.

INDEX

Keywords : appellativity, insult, interdiscourse, interpellation, speech acts, text, typology Mots-clés : appellativité, injure, interaction verbale, interdiscours, interpellation, typologie textuelle

\section{AUTEUR}

\section{FRANÇOISE HAMMER}

Universität Karlsruhe 